\title{
Tinkering with chromatin
}

Protein trans-splicing is used to study chromatin signaling in the cell nucleus.

A growing arsenal of chemical tools is enabling researchers to perturb and observe biological molecules in the cell. Inteins are naturally occurring protein segments that excise themselves in the process of protein splicing-a useful property that chemists have exploited. In the technique of protein trans-splicing, two polypeptides are each fused to one half of a split intein, which joins the fused segments via a peptide bond during splicing. This technology can be used in cells, it enables a broad range of modifications to be installed onto a protein of interest, and it is traceless-meaning that no trace of the intein remains in the final spliced product.

Tom Muir of Princeton University has been developing and utilizing intein chemistry for many years. In recent work, his group applied protein trans-splicing to a challenging biological problem: studying chromatin regulation in the nucleus of a cell.

Muir's team identified a split intein pair with fast and efficient splicing: an N-terminal intein fragment from Anabaena variabilis $\left(\right.$ Ava $\left.^{\mathrm{N}}\right)$ and a C-terminal intein fragment from Nostoc punctiforme $\left(\mathrm{Npu}^{\mathrm{C}}\right)$. They expressed histone $\mathrm{H} 2 \mathrm{~B}-\mathrm{Ava}^{\mathrm{N}}$ in the cell and used a cell-penetrating peptide to deliver a construct consisting of $\mathrm{Npu}^{\mathrm{C}}$ fused to the epitope tag HA (human influenza hemagglutinin). They confirmed the expected H2BHA splice product with western blotting and mass spectrometry.

Confident that the splicing system was working as planned, the researchers next used it to probe chromatin structure in isolated nuclei. They selected various genes known to reside in either euchromatin or heterochromatin and assessed the rate of $\mathrm{H} 2 \mathrm{~B}-\mathrm{HA}$ splice product formation at these loci using chromatin immunoprecipitation and quantitative PCR. This analysis revealed that the more loosely packed euchromatic regions were much more reactive than the tightly packed heterochromatic regions, as expected.

Finally, the team used their system to install ubiquitin onto lysine 120 (K120) of $\mathrm{H} 2 \mathrm{~B}$. This allowed them to investigate the suspected cross-talk of this modification with $\mathrm{H} 3 \mathrm{~K} 79$ methylation in the cell nucleus. Upon generating the $\mathrm{H} 2 \mathrm{~B}-\mathrm{K} 120 \mathrm{ub}$ product, they observed a twofold increase in H3K79 methylation levels, thereby showing that the ubiquitin induces a chromatin signaling event.

The approach will undoubtedly be useful for tinkering with chromatin to address a number of open questions in epigenetics.

\section{Allison Doerr}

\section{RESEARCH PAPERS}

David, Y. et al. Chemical tagging and customizing of cellular chromatin states using ultrafast transsplicing inteins. Nat. Chem. 7, 394-402 (2015). 